Research Article

\title{
Electromagnetic Vector Sparse Nested Array: Array Structure Design, Off-Grid Parameter Estimation Algorithm
}

\author{
Beizuo Zhu $\mathbb{D}^{1,2}$ Weiyang Chen $\mathbb{D}^{1,2}$ and Luo Chen ${ }^{1,2,3}$ \\ ${ }^{1}$ College of Electronic and Information Engineering, Nanjing University of Aeronautics and Astronautics, Nanjing 211106, China \\ ${ }^{2}$ Key Laboratory of Dynamic Cognitive System of Electromagnetic Spectrum Space (Nanjing University of Aeronautics and \\ Astronautics), Ministry of Industry and Information Technology, Nanjing 211106, China \\ ${ }^{3}$ The $28^{\text {th }}$ Research Institute of China Electronics Technology Group Corporation, Nanjing 210007, China \\ Correspondence should be addressed to Weiyang Chen; chenweiyang2020@126.com
}

Received 6 January 2021; Revised 15 February 2021; Accepted 15 March 2021; Published 27 March 2021

Academic Editor: Junpeng Shi

Copyright (C) 2021 Beizuo Zhu et al. This is an open access article distributed under the Creative Commons Attribution License, which permits unrestricted use, distribution, and reproduction in any medium, provided the original work is properly cited.

\begin{abstract}
In this paper, a new array structure of sparse nested array (SNA) for electromagnetic vector sensor is designed. An electromagnetic vector sensor is composed of six spatially colocated, orthogonally oriented, diversely polarized antennas, which can measure three-dimensional electric and magnetic field components. By introducing sparse factor (SF) between every adjacent sensor, the proposed SNA has flexibility of extending the array aperture and reducing the mutual coupling effect. Meanwhile, a low-complexity multiparameter estimation algorithm is proposed for SNA. First, the vectorization operation for array manifold ensures the large degrees of freedom for multiparameter estimation, where the initial coarse estimates decrease search range. In addition, the improved off-grid orthogonal matching pursuit method obtains joint direction of arrival (DOA) and polarization estimates with a relatively small overcomplete dictionary because this off-grid method achieves high performance even if the estimates do not fall on the grid of the dictionary. Theoretical analysis and simulation results verify the superiority of the proposed array structure and the algorithm.
\end{abstract}

\section{Introduction}

Vector sensors, which are able to detect multiple physical components of the signals, have been widely used in array signal processing [1-3]. Compared with scalar sensor arrays, vector sensor arrays show their advantages in estimation accuracy, recognition accuracy, and antijamming capability [4-6]. Moreover, vector sensor arrays can obtain joint estimates of multiple parameters, such as electromagnetic vector sensor array (EVSA). EVSA can measure DOA and polarization information at the same time because vector sensor structure has the reception access of vector signals.

Resultantly, various DOA and polarization estimation algorithms are proposed for EVSAs, where most of them are inspired by the algorithms for scalar arrays. For example, ESPRIT- (Estimating Signal Parameter via Rotational Invariance Techniques-) based algorithm is proposed in [7, 8], estimating both the arrival angles and the polarizations of incoming narrow-band signals with invariance properties of the EVSA. MUSIC (Multiple Signal Classification) algorithm is also transformed for EVSA in [9], where the joint DOA and polarization estimates are measured by peak search. For alleviating the high computational burden in peak search, a reduced-dimensional MUSIC algorithm is put forward [10], where only two-dimensional peak search is necessary for 4 unknown parameters. In addition, [11] proposes a novel rank reduction method for DOA, range, and polarization estimation, but near-field signal hypothesis is limited.

Meanwhile, some other studies concentrate on the improvement of array structures for EVSAs. The researched algorithms are mainly based on half-wavelength interval arrays, where the array aperture is restricted by the number of sensors and mutual coupling effect has an adverse impact on array performance. Moreover, this kind of array structures has the number of degrees of freedom (DOFs) less than 
the number of physical sensors, which means that algorithms cannot work when signal numbers are more than sensor numbers. To track the problems, sparse arrays are presented in polarization environment to avoid compact placement and increase the number of DOFs [12-18]. A series of vector cross-product-based algorithms are introduced in [12-14]. This kind of algorithms extracts DOA parameters by performing cross-product to Poynting vector in received signal, which first breaks the limitation of halfwavelength intervals for EVSA. Variable separation MUSIC/ MODE algorithm [15] achieves the unambiguous search results for direction of arrivals, which is capable for sparse uniform array structures, but polarization estimation is ignored. The study in [16] applies coprime array in polarization sensors, obtaining joint DOA and polarization estimates with compressed sensing reconstruction algorithm. Due to the vectorization operation for array manifold, the number of DOFs is tremendously increased. In [18], sparse representation (SR) idea is taken for three-parallel coprime EVSA. However, all aforementioned papers focus on the specific array structures and computational burden is relatively high, which is not flexible for different actual engineering requirements.

In this paper, we propose a flexible array structure called sparse nested array (SNA), which can be considered as an improvement of traditional nested array (NA). To be specific, every sensor is equipped with six spatially colocated, orthogonally oriented, diversely polarized antennas, where three cocentered orthogonal electric dipoles and magnetic loops are included. The proposed SNA enjoys flexible sensor interval benefitting from sparse factor (SF) $\delta \geq 1$. The subarrays 1 and 2 in SNA are both uniform linear arrays composed of $M$ and $N$ sensors with intervals $\delta \lambda / 2(M+1) \delta \lambda / 2$, where $\lambda$ is the wavelength. The interval between the two subarrays is also $(M+1) \delta \lambda / 2$. SF is a positive integer to adjust spacing between sensors overall. By the enlargement of the array aperture, estimation performance is improved and mutual coupling effect is alleviated.

Meanwhile, from the perspective of algorithm, cross-product of the Poynting vector is employed as the coarse DOA initialization. In addition, after using the properties of the covariance matrix to eliminate the polarization parameters, vectorization operation is taken to construct virtual uniform array, which brings about large DOFs of $O(M N)$ with $M+N$ sensors. Then we apply the off-grid orthogonal matching pursuit (OGOMP) algorithm to obtain high-precision multiparameter estimation. Computational complexity is tremendously alleviated because the one-dimensional overcomplete dictionary in OGOMP algorithm is established only around initial DOA estimates. Traditional OMP algorithm [19] requires that all target signals must fall on a preset grid. However, in actual engineering applications, no matter how the grid is divided, it is impossible to ensure that all target signals fall exactly on the grid. When the target signal is off-grid, the estimation performance of the system will be greatly reduced. On the other hand, if the grid is divided too finely, it will cause the system to have too much calculation burden.
Moreover, there is not any ambiguous or pairing problem disturbing true values because OGOMP is an ambiguityfree autopaired algorithm.

In short, we summarize the innovations of this paper as follows:

(1) We design a new structure of nested array in EVSA, where six-component electromagnetic vector sensors are equipped, extending array aperture as well as reducing mutual coupling effect. By the vectorization operation of the manifold, high DOFs can be obtained.

(2) We add sparse factor (SF) in every interval of NA, constructing a new array structure called sparse nested array (SNA), which enjoys scaled array aperture and adjustable mutual coupling effect. Meanwhile, the proposed array can maintain the uniqueness of parameter estimates, which aims to be suitable for different engineering scenarios.

(3) We propose a low-complexity off-grid OMP (OGOMP) algorithm to measure joint DOA and polarization estimates. Combined with the off-grid idea that the target signals do not need to just fall on the grid, OGOMP algorithm can use much smaller one-dimensional overcomplete dictionary around initial DOA estimates, tremendously alleviating computational burden as well as performing good estimation performance.

Notations. We use lower-case (upper-case) bold character to denote vector (matrix). $(\cdot)^{*},(\cdot)^{T}$, and $(\cdot)^{H}$ are the conjugate, transpose, and conjugate transpose of a matrix or vector, respectively. $(\cdot)^{-1}$ denotes matrix inverse and $(\cdot)^{+}$ denotes matrix pseudoinverse. $\oplus$ represents Hadamard product. $\otimes$ denotes the Kronecker product and $\odot$ represents the Khatri-Rao product. $\operatorname{diag}(\cdot)$ symbolizes a diagonal matrix that uses the elements of the matrix as its diagonal element. abs(.) is absolute value operator and angle $(\cdot)$ is phase operator. $\mid \cdot \|_{1}$ denotes 1 norm and $\|\cdot\|_{F}$ denotes Frobenius norm.

\section{Preliminaries}

2.1. Data Model. Consider an array with a certain amount of electromagnetic vector sensors and every sensor herein is equipped with three cocentered orthogonal electric dipoles and magnetic loops, which is shown in Figure 1 [20].

Assume that there are $K$ far-field narrow-band signals impinging on the array with $P$ electromagnetic vector sensors distributed at $y$-axis with $D_{p}=d_{0} d_{p}, p=1,2$, $\ldots, P$, which is demonstrated in Figure 2 .

$d_{0}=\lambda / 2$ is the unit spacing between adjacent sensors, $d_{p} \in \mathbb{Z}$, and $\lambda$ symbolizes the wavelength. The $K$ signals are all completely polarized from yoz plane with incidence angles $\theta_{k}, k=1,2, \ldots, K$. The three electric components and magnetic components of the $k$-th signal at $x, y, z$-axes are received by the loops and dipoles, which can be represented as [21] 


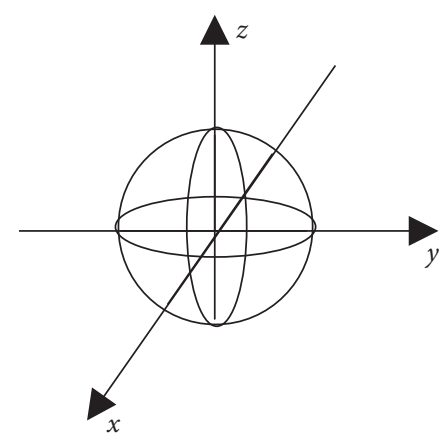

FIGURE 1: Internal structure of sensor element.

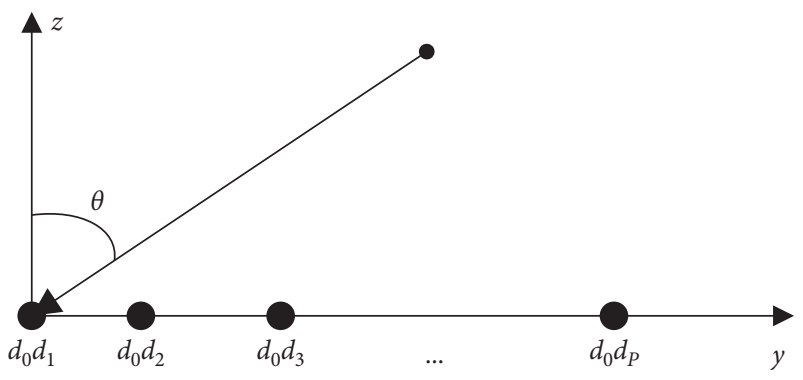

Figure 2: Array model.

$$
\mathbf{s}_{k}=\left[\begin{array}{c}
\mathbf{s}_{e x, k} \\
\mathbf{s}_{e y, k} \\
\mathbf{s}_{e z, k} \\
\mathbf{s}_{h x, k} \\
\mathbf{s}_{h y, k} \\
\mathbf{s}_{h z, k}
\end{array}\right]=\left[\begin{array}{cc}
0 & -1 \\
\cos \theta_{k} & 0 \\
-\sin \theta_{k} & 0 \\
-1 & 0 \\
0 & -\cos \theta_{k} \\
0 & \sin \theta_{k}
\end{array}\right]\left[\begin{array}{c}
\sin \gamma_{k} e^{j \eta_{k}} \\
\cos \gamma_{k}
\end{array}\right]=\boldsymbol{\Phi}_{k} \boldsymbol{\omega}_{k}
$$

where $\mathbf{s}_{e x, k}, \mathbf{s}_{e y, k}, \mathbf{s}_{e z, k}$ denote the electric components and $\mathbf{s}_{h x, k}, \mathbf{s}_{h y, k}, \mathbf{s}_{h z, k}$ denote the magnetic components of the loops and dipoles. $\mathbf{s}_{e, k}=\left[\mathbf{s}_{e x, k}, \mathbf{s}_{e y, k}, \mathbf{s}_{e z, k}\right]$ and $\mathbf{s}_{h, k}=\left[\mathbf{s}_{h x, k}, \mathbf{s}_{h y, k}\right.$, $\left.\mathbf{s}_{h z, k}\right]$ are orthogonal to each other and also to the $k$-th source's direction of propagation. $\gamma_{k} \in[0, \pi / 2]$ is the auxiliary polarization angle and $\eta_{k} \in[-\pi, \pi)$ represents the polarization phase difference, respectively. Resultantly, the data model of the received signal at $t$ time can be expressed as [17]

$$
\mathbf{x}(t)=(\mathbf{A} \odot \mathbf{S}) \mathbf{b}(t)+\mathbf{n}(t)=\mathbf{A}_{s} \mathbf{b}(t)+\mathbf{n}(t),
$$

where $\mathbf{A}=\left[\mathbf{a}_{1}, \mathbf{a}_{2}, \ldots, \mathbf{a}_{K}\right]$ is the directional matrix and $\mathrm{a}_{k}=$ $\left[e^{j D_{1} 2 \pi \sin \theta_{k} / \lambda}, \ldots, e^{j D_{P} 2 \pi \sin \theta_{k} / \lambda}\right]$ denotes the directional vector for $k$ - th signal containing DOA information. $\mathbf{S}_{k}=$ $\left[\mathbf{s}_{1}, \mathbf{s}_{2}, \ldots, \mathbf{s}_{K}\right]$ is the polarization vector matrix, $\mathbf{b}(t) \in C^{J \times 1}$ symbolizes the signal vector, and $\mathbf{n}(t)$ denotes the additive white Gaussian noise complex vector. $\odot$ represents the Khatri-Rao product.

Construct the covariance matrix:

$$
\mathbf{R}_{s}=E\left[\mathbf{x}(t) \mathbf{x}^{H}(t)\right]=\mathbf{A} \odot \mathbf{S}\left[\operatorname{diag}\left(\sigma_{1}^{2}, \sigma_{2}^{2}, \ldots, \sigma_{K}^{2}\right)\right](\mathbf{A} \odot \mathbf{S})^{H},
$$

where $E[\cdot]$ denotes the expectation operation. In practice, snapshots $J$ received are finite and they can be approximately calculated by

$$
\mathbf{R}_{s} \approx \frac{1}{J} \sum_{j=1}^{J} \mathbf{x}(t) \mathbf{x}^{H}(t) .
$$

Meanwhile, it is also recognized that we can reconstruct the covariance matrix separately according to the six-component received electric and magnetic signals.

$$
\begin{aligned}
& \left\{\begin{array}{l}
\mathbf{R}_{e x}=\operatorname{Adiag}\left(\sigma_{1}^{2} \mathbf{s}_{e x, 1} \mathbf{s}_{e x, 1}^{*}, \sigma_{2}^{2} \mathbf{s}_{e x, 2} \mathbf{s}_{e x, 2}^{*}, \ldots, \sigma_{K}^{2} \mathbf{s}_{e x, K} \mathbf{s}_{e x, K}^{*}\right) \mathbf{A}^{H} \\
\mathbf{R}_{e y}=\operatorname{Adiag}\left(\sigma_{1}^{2} \mathbf{s}_{e y, 1} \mathbf{s}_{e y, 1}^{*}, \sigma_{2}^{2} \mathbf{s}_{e y, 2} \mathbf{s}_{e y, 2}^{*}, \ldots, \sigma_{K}^{2} \mathbf{s}_{e y, K} \mathbf{s}_{e y, K}^{*}\right) \mathbf{A}^{H}, \\
\mathbf{R}_{e z}=\operatorname{Adiag}\left(\sigma_{1}^{2} \mathbf{s}_{e z, 1} \mathbf{s}_{e z, 1}^{*}, \sigma_{2}^{2} \mathbf{s}_{e z, 2} \mathbf{s}_{e z, 2}^{*}, \ldots, \sigma_{K}^{2} \mathbf{s}_{e z, K} \mathbf{s}_{e z, K}^{*}\right) \mathbf{A}^{H}
\end{array}\right. \\
& \left\{\begin{array}{l}
\mathbf{R}_{h x}=\operatorname{Adiag}\left(\sigma_{1}^{2} \mathbf{s}_{h x, 1} \mathbf{s}_{h x, 1}^{*}, \sigma_{2}^{2} \mathbf{s}_{h x, 2} \mathbf{s}_{h x, 2}^{*}, \ldots, \sigma_{K}^{2} \mathbf{s}_{h x, K} \mathbf{s}_{h x, K}^{*}\right) \mathbf{A}^{H} \\
\mathbf{R}_{h y}=\operatorname{Adiag}\left(\sigma_{1}^{2} \mathbf{s}_{h y, 1} \mathbf{s}_{h y, 1}^{*}, \sigma_{2}^{2} \mathbf{s}_{h y, 2} \mathbf{s}_{h y, 2}^{*}, \ldots, \sigma_{K}^{2} \mathbf{s}_{h y, K} \mathbf{s}_{h y, K}^{*}\right) \mathbf{A}^{H}, \\
\mathbf{R}_{h z}=\operatorname{Adiag}\left(\sigma_{1}^{2} \mathbf{s}_{h z, 1} \mathbf{s}_{h z, 1}^{*}, \sigma_{2}^{2} \mathbf{s}_{h z, 2} \mathbf{s}_{h z, 2}^{*}, \ldots, \sigma_{K}^{2} \mathbf{s}_{h z, K} \mathbf{s}_{h z, K}^{*}\right) \mathbf{A}^{H}
\end{array}\right.
\end{aligned}
$$

where $\sigma_{k}^{2}, k=1,2, \ldots, K$ denotes the power of the $k$-th signal. By splitting $\mathbf{S}$ and calculating the covariance matrix individually for the six components, we put the polarization information into diag function. Note that

$$
\begin{aligned}
& \mathbf{s}_{e x, k} \mathbf{s}_{e x, k}^{*}+\mathbf{s}_{e y, k} \mathbf{s}_{e y, k}^{*}+\mathbf{s}_{e z, k} \mathbf{s}_{e z, k}^{*}=1, \\
& \mathbf{s}_{h x, k} \mathbf{s}_{h x, k}^{*}+\mathbf{s}_{h y, k} \mathbf{s}_{h y, k}^{*}+\mathbf{s}_{h z, k} \mathbf{s}_{h z, k}^{*}=1 .
\end{aligned}
$$

Consequently, the covariance matrix without polarization information can be obtained.

$$
\begin{aligned}
\mathbf{R} & =\mathbf{R}_{e x}+\mathbf{R}_{e y}+\mathbf{R}_{e z}=\mathbf{R}_{h x}+\mathbf{R}_{h y}+\mathbf{R}_{h z}, \\
& =\operatorname{Adiag}\left(\sigma_{1}^{2}, \cdots, \sigma_{K}^{2}\right) \mathbf{A}^{H} .
\end{aligned}
$$

2.2. Mutual Coupling. The data model established in Section 2.1 is in the case of free mutual coupling. In actual engineering, there might be serious mutual coupling effect between sensors, especially in adjacent sensors close to each other. The data model considering the influence of mutual coupling is expressed as [22]

$$
\mathbf{Y}=[(\mathbf{C A}) \odot \mathbf{S}] \mathbf{B}^{T}+\mathbf{N},
$$

where $\mathbf{C}$ is a $P \times P$ matrix reflecting interelement coupling (IEC), which is determined by the array manifold. $\mathbf{C}$ can be established according to different criteria. In this paper, B-banded mutual coupling model is employed based on Toeplitz property. Resultantly, mutual coupling matrix $\mathbf{C}$ is defined as 


$$
\mathbf{C}(i, j)=\left\{\begin{array}{ll}
0 & \left|d_{i}-d_{j}\right|>B \\
c_{\mid d_{i}-d_{j}} \mid & \left|d_{i}-d_{j}\right| \leq B
\end{array},\right.
$$

where $c_{n}=c_{1} e^{-j(n-1) \pi / 8} / n,(2 \leq n \leq B)$ and $c_{1}$ is the basic mutual coupling strength with sensor intervals $d_{0}=\lambda / 2$. $d_{i}, d_{j},(1 \leq i \leq P, 1 \leq j \leq P)$ denote the position of the sensor elements. $B$ represents the maximum distance in which mutual coupling takes effect among sensors. Due to the introduction of mutual coupling matrix, a standard of coupling leakage $(\Gamma)$ can be set for judging the strength of mutual coupling.

$$
\Gamma=\frac{\|\mathbf{C}-\operatorname{diag}(\mathbf{C})\|_{F}}{\|\mathbf{C}\|_{F}},
$$

where $\mid \cdot \|_{F}$ denotes Frobenius norm.

Remark 1. Because three orthogonal electric dipoles and magnetic loops in an electromagnetic sensor are designed as a whole part, the interpolarization coupling (IPC) can be measured in application. In this case, we eliminate the influence of IPC in received signal model and only consider the effect of IEC.

\section{Array Structure Design}

3.1. Sparse Nested Array. The structure of sparse nested array (SNA) is presented in Figure 3. The first subarray, which is marked by black circles, is a uniform linear array with $M$ sensors. The internal spacing between adjacent sensors is $\delta d_{0}$, where $\delta \in \mathbb{N}^{+}$is named as sparse factor. The second subarray marked with white squares contains $N$ sensors, which is also a uniform linear array whose interval between sensors is $(M+1) \delta d_{0}$. The total numbers of sensors are $M+N=P$. Both subarray 1 and subarray 2 lie on $y$-axis and the $M$-th sensor in subarray 1 and the first sensor in subarray 2 have the $\delta d_{0}$ interval.

Compared with traditional NA [23], the proposed SNA is developed by the sparse factor $\delta$ to unfold sensor interval. It is indicated in Figure 3 that when $\delta=1$, NA is a special case of SNA.

3.2. Interpolation for Virtual Array. According to the basic knowledge of array signal processing, $P$ sensors can achieve $P-1$ degrees of freedom (DOFs). Nevertheless, some sparse arrays can further enlarge DOFs by their equivalent virtual arrays and perform estimation algorithm with the reconstructed virtual signals. In this paper, difference coarray is employed to obtain virtual array of SNA. According to (8), reconstructed covariance matrix $\mathbf{R}$ is a $P \times P$ matrix with DOA information received by the array. By vectorizing the covariance matrix, the equivalent virtual array signals are expressed as [24]

$$
\mathbf{z}=\operatorname{vec}(\mathbf{R})=\left(\mathbf{A}^{*} \odot \mathbf{A}\right) \mathbf{b}_{s}^{T},
$$

where $\mathbf{A}^{*} \odot \mathbf{A}=\left[\mathbf{a}_{1}^{*} \otimes \mathbf{a}_{1}, \mathbf{a}_{2}^{*} \otimes \mathbf{a}_{2}, \ldots, \mathbf{a}_{K}^{*} \otimes \mathbf{a}_{\mathbf{K}}\right] \in \mathbb{C}^{P^{2} \times K}$ denotes the virtual directional matrix. $\mathbf{b}_{s}=\left[\sigma_{1}^{2}, \ldots, \sigma_{K}^{2}\right]$ is the

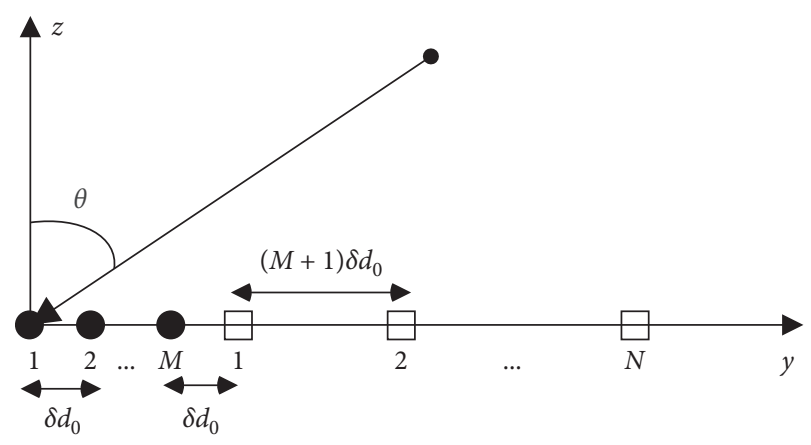

FIgURE 3: Structure of sparse nested array.

equivalent one-snapshot signal vector. We can find that the position of virtual sensor element is located at $D \delta$, where

$$
D=\left\{d_{0}\left(d_{i}-d_{j}\right) \mid i, j=1,2, \ldots, P\right\} .
$$

Obviously, there exist repeated elements in set $D$. By removing these elements, a unique subset $D_{u}$ is established. The virtual location of the received signal after vectorization is modeled as

$$
D_{u}=\left\{d_{0} d_{u} \mid-[N \times(M+1)-1] \leq d_{u} \leq[N \times(M+1)-1], d_{u} \in \mathbb{Z}\right\} .
$$

Consequently, only the data of $\left|D_{u}\right|=2(N \times(M+1)-$ $1)+1$ rows is necessary for DOA estimation. By selecting the corresponding rows in $\mathbf{z}$, the reconstructed virtual received signals are built using the full DOFs.

$$
\mathbf{z}_{u}=\mathbf{A}_{u} \mathbf{b}_{s}^{T},
$$

where $\mathbf{A}_{u}=\left[\mathbf{a}_{u}\left(\theta_{1}\right), \mathbf{a}_{u}\left(\theta_{2}\right), \ldots \mathbf{a}_{u}\left(\theta_{K}\right)\right] \in \mathbb{C}^{\left|D_{u}\right| \times K}$ is the equivalent virtual array located at $D_{u} \delta$, which is a uniform linear array, and every adjacent virtual sensor has the interval of $\delta d_{0}$.

\subsection{Discussion}

3.3.1. Virtual Array Configuration. Compared with coprime array, nested array is a kind of completely augmented array whose virtual array is continuous without holes. Define unit length as half wavelength $\lambda / 2$. In order to give an intuitive understanding, we demonstrate the virtual sensor elements of typical coprime array $M=5, N=4$ and nested array $M=N=4$, where $\delta=1$. Both NA and CA have 8 physical sensors because there is a shared sensor for CA. Black circles and white squares denote the physical sensors belonging to the first and second subarrays, respectively. The black crosses represent the virtual sensor elements. From the comparison of Figures 4 and 5, NA has 39 virtual sensor elements and all of them are continuous without holes. Meanwhile, CA only has 27 virtual sensor elements and there are 6 missing elements in $\{-14,-13,-9,9,13,14\}$, which are marked by red crosses. As a result, CA is unable to make full use of information on the whole virtual array, acquiring fewer DOFs and smaller array aperture than NA. 


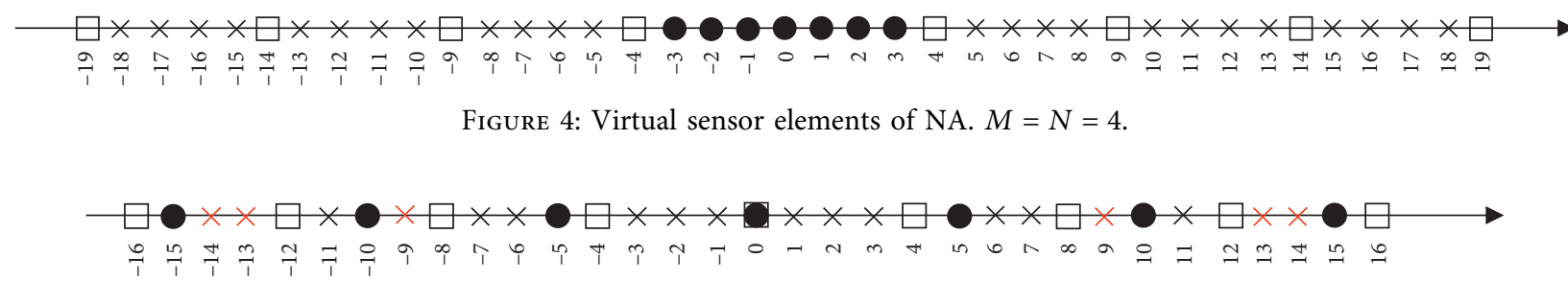

Figure 5: Virtual sensor elements of CA. $M=5, N=4$.

Definition 1 (array aperture). Array aperture is defined as the length of the total linear array, which is one of the criteria evaluating array performance.

3.3.2. Engineering Problems of Sparse Array. According to Section 2, mutual coupling effect decreases exponentially along with the sensor interval. Resultantly, small intervals should be prevented possibly. Sparse arrays, due to the inherent merits of loose array structure, have much lower mutual coupling effect than traditional arrays with halfwavelength intervals. The proposed SNA in this paper solves the problems of compact array structure of NA in the first subarray, as well as providing a relatively flexible array configuration. Moreover, with the increase of sparse factor $\delta$, mutual coupling effect can be further alleviated.

Notation. Although sparse factor provides many gains in array performance, it is not an unlimited number in practice. The three main factors limiting sparse factor are array decorrelation, far-field hypothesis, and grid misidentification. The first two factors are due to actual engineering and the third is due to algorithm limitation. We discuss the factors, respectively, in the following part.

Array decorrelation: suppose that a narrow-band signal is expressed as

$$
s(t)=a(t) e^{j\left[\omega_{0} t+\phi(t)\right]},
$$

where $a(t)$ denotes the slowly varying amplitude modulation function, which is considered abiding during signal reception time, $\omega_{0}=2 \pi f_{0}$ is the carrier frequency, and $\phi(t)$ represents the phase modulation function. Mark the received signal on the array as $\left[s\left(t_{1}\right), s\left(t_{2}\right), \ldots, s\left(t_{P}\right)\right]$. The amplitude modulation function must guarantee

$$
[\max |a(t)|-\min |a(t)|] / \max |a(t)|<q,
$$

where $t \in\left[t_{1}, t_{2}, \ldots, t_{P}\right]$ and $0<q<1$ depends on actual needs. With the increase of sparse factor, array decorrelation occurs, which will tremendously affect estimation performance.

Far-field hypothesis: we assume that all signals are all from the far field, which satisfy the condition that

$$
Z \geq \frac{2 \varsigma^{2}}{\lambda}
$$

where $Z$ denotes the minimum distance from any signal to the array. $\varsigma$ represents the array aperture.

Grid misidentification: this problem often occurs in low signal-to-noise ratio (SNR) environments because the noise generates a much larger phase shift in directional matrix, which may undermine the orthogonality of search algorithms [25]. On the other hand, ESPRIT-based algorithms need ambiguity elimination operations. Arrays with large sparse factor have small grids, which leads to closer ambiguous values. In low SNR, it is easy to mismatch with wrong estimates.

3.4. Performance Analysis. For intuitive comparison, Table 1 lists the DOFs after vectorization operation, array aperture, and mutual coupling effect for SNA, SCA, and traditional uniform linear array (TULA) [26] with all 8 physical sensors. For simplicity, label $\lfloor a, b, c, d, e\rfloor$ is utilized to indicate that subarray 1 has $a$ sensors with $b \delta d_{0}$ intervals and subarray 2 has $c$ sensors with $d \delta d_{0}$ intervals and $e$ denotes the number of sparse factors $\delta$.

It is revealed in Table 1 that both nested array and coprime array outperform traditional uniform linear array. By enlarging sparse factor or increasing the number of physical sensors, SNA and SCA achieve larger array aperture and lower mutual coupling. Meanwhile, NA has more DOFs and extended array aperture than CA with the same physical sensors.

\section{DOA and Polarization Estimation Algorithm}

Orthogonal matching pursuit (OMP) algorithm is considered as a typical compressed sensing method to obtain DOA estimates. However, an overcomplete dictionary is necessary for orthogonal verification, which takes relatively high computational complexity. Moreover, orthogonal verification is essentially a searching process, where grid density affects both estimation accuracy and computational burden. The two indexes check and balance with each other. Off-grid orthogonal matching pursuit (OGOMP) solves off-grid problem and guarantees good performance. Based on the scalar OGOMP algorithm [27], we propose a lowcomplexity DOA and polarization estimation algorithm for SNA, which mainly includes DOA initialization and accurate DOA and polarization estimation.

4.1. Initial DOA Estimation. According to the covariance matrix $\mathbf{R}_{s}$, eigendecomposition can be performed to obtain signal subspace $\mathbf{E}_{s} \in \mathbb{C}^{6(M+N) \times K}$. On the other hand, the first to $(M-1)$-th sensors have rotation invariance with the second to $M$-th sensors in subarray 1 and the first to $(N-1)$-th sensors have rotation invariance with the second to $N$-th sensors in subarray 2. Therefore, we can decompose the signal subspace $\mathbf{E}_{s}$. 


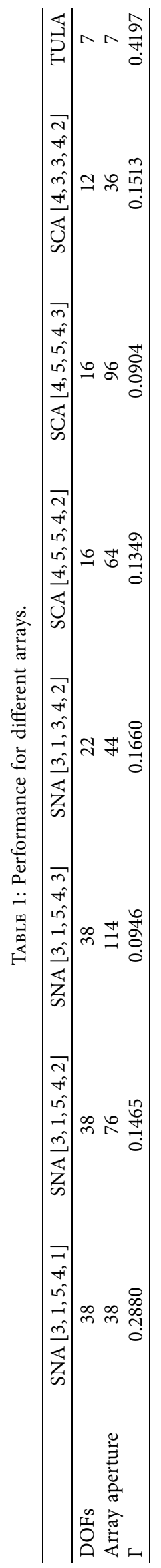




$$
\begin{aligned}
& \mathbf{E}_{s 1}=\mathbf{E}_{s}[1: 6(M-1)], \\
& \mathbf{E}_{s 2}=\mathbf{E}_{s}[7: 6 M], \\
& \mathbf{E}_{s 3}=\mathbf{E}_{s}[6 M+1: 6(M+N-1)], \\
& \mathbf{E}_{s 4}=\mathbf{E}_{s}[6 M+7: 6(M+N)],
\end{aligned}
$$

where $\mathbf{E}_{s}[a: b]$ represents the line $a$ to $b$ of $\mathbf{E}_{s}$. By eigendecomposition of $\mathbf{E}_{s 1}{ }^{+} \mathbf{E}_{s 2}$ and $\mathbf{E}_{s 3}{ }^{+} \mathbf{E}_{s 4}$, eigenvectors $\mathbf{T}_{12}, \mathbf{T}_{34}$ and eigenvalues $\mathbf{v}_{12}=\left[e^{j \delta d_{0} 2 \pi \sin \theta_{1} / \lambda}, \ldots, e^{j \delta d_{0} 2 \pi \sin \theta_{K} / \lambda}\right]$ and $\mathbf{v}_{34}=\left[e^{j(M+1) \delta d_{0} 2 \pi \sin \theta_{1} / \lambda}, \ldots, e^{j(M+1) \delta d_{0} 2 \pi \sin \theta_{K} / \lambda}\right]$ can be calculated, where the eigenvectors are nonsingular $K \times K$ matrices with full rank. We employ a vital characteristic in array signal processing [7].

$$
\mathbf{E}_{s}=\mathbf{A}_{s} \mathbf{T}
$$

Thus, the estimate $\mathbf{A}_{s}$ is measured by

$$
\mathbf{A}_{s}=\left\{\begin{array}{c}
\mathbf{E}_{s}[1: 6 M] \mathbf{T}_{12}^{-1} \\
\mathbf{E}_{s}[6 M+1: 6(M+N)] \mathrm{T}_{34}^{-1}
\end{array}\right\} .
$$

The next step is extracting the DOA parameters from $\mathbf{A}_{s}=\left[\mathbf{a}_{1} \otimes \mathbf{s}_{1}, \mathbf{a}_{2} \otimes \mathbf{s}_{2}, \ldots, \mathbf{a}_{K} \otimes_{K}\right]$ as the initial estimation results. Here, we eliminate the directional matrix $\mathbf{A}=\left[\mathbf{a}_{1}, \mathbf{a}_{2}, \ldots, \mathbf{a}_{K}\right]$ by $\mathbf{v}_{12}, \mathbf{v}_{34}$ to estimate the polarization vector matrix $\widehat{S}=\left[\mathbf{s}_{1}, \mathbf{s}_{2}, \ldots, \mathbf{s}_{K}\right]$, which is expressed as

$$
\begin{aligned}
\mathbf{s}_{k}= & \frac{1}{2 M} \sum_{i=1}^{M} \frac{\mathbf{A}_{s, k}[6(i-1)+1: 6 i]}{\| \mathbf{A}_{s, k}\left[6(i-1)+1: 6 i \|_{1}\right.}\left(\mathbf{v}_{12}^{[k]}\right)^{-j(i-1)} \\
& +\frac{1}{2 N} \sum_{i=1}^{N} \frac{\mathbf{A}_{s, k}[6 M+6(i-1)+1: 6 M+6 i]}{\| \mathbf{A}_{s, k}\left[6 M+6(i-1)+1: 6 M+6 i \|_{1}\right.}\left(\mathbf{v}_{34}^{[k]}\right)^{-j(i-1)},
\end{aligned}
$$

where $\mathbf{A}_{s, k}$ denotes the $k$ - th row of $\mathbf{A}_{s, k}$ and $\mathbf{v}^{[k]}$ is the $k$-th element of $\mathbf{v}$. As is revealed in (22), each term on the right side of the equation is an estimate of $\mathbf{s}_{k}$, which uses up the full information of $\mathbf{A}_{s}$ to get more precise results.

According to (1), the normalized Poynting vector $\mathbf{P}_{k}$ can be estimated with vector cross-product estimator, which is expressed as [15]

$$
\mathbf{P}_{k}=\left[\begin{array}{l}
\mathbf{P}_{x, k} \\
\mathbf{P}_{y, k} \\
\mathbf{P}_{z, k}
\end{array}\right]=\mathbf{s}_{e, k} \times \mathbf{s}_{h, k}=\left[\begin{array}{c}
0 \\
\sin \theta \\
\cos \theta
\end{array}\right],
$$

where only DOA information is involved in $\mathbf{P}_{k}$. Based on the analysis above, we can obtain the coarse initial DOA estimates.

$$
\widehat{\theta}_{k, \text { ini }}=\frac{1}{2}\left[\arcsin \mathbf{P}_{k}^{[2]}+\arccos \mathbf{P}_{k}^{[3]}\right],
$$

where $\mathbf{P}^{[i]}$ denotes the $i$-th element of $\mathbf{P}$.

4.2. Precise DOA Estimation with Low Complexity. In this part, we propose an off-grid OMP (OGOMP) algorithm, which can obtain accurate joint DOA and polarization estimates.
First, we can establish an overcomplete dictionary partly taken from $\mathbf{A}_{u}$ :

$$
\mathbf{Q}(\theta)=\left[\mathbf{a}_{u}\left(\theta_{1}\right), \mathbf{a}_{u}\left(\theta_{2}\right), \ldots, \mathbf{a}_{u}\left(\theta_{Q}\right)\right] \in \mathbb{C}^{\left|D_{u}\right| \times Q}, Q \gg K,
$$

where the angular interval is $r=\theta_{i+1}-\theta_{i}, 1 \leq i \leq Q-1$ and $\theta_{i}$ is near the initial DOA estimates. Define a deviation vector $\xi \in \mathbb{R}^{Q \times 1}$ and every element $-r / 2 \leq \xi_{q} \leq r / 2, q=1,2, \ldots, Q$ refers to the deviation with grid. The directional vector after grid division will be close to the directional vector of the actual target signal with the first-order Taylor expansion principle, which can be expressed as

$$
\mathbf{a}_{u t}\left(\theta_{i}+\xi_{i}\right) \approx \mathbf{a}_{u}\left(\theta_{i}\right)+\frac{\partial \mathbf{a}_{u}\left(\theta_{i}\right)}{\partial \theta_{i}} \xi_{i}, \quad i=1,2, \ldots, Q,
$$

where $\mathbf{a}_{u}\left(\theta_{i}\right)$ denotes the directional vector of $\theta_{i}$ in overcomplete dictionary corresponding to virtual array manifold after vectorization and $\partial($.$) represents the partial derivative.$ Therefore,

$\mathbf{Q}_{t}=\left[\mathbf{a}_{u t}\left(\theta_{1}+\xi_{1}\right), \mathbf{a}_{u t}\left(\theta_{2}+\xi_{2}\right), \ldots, \mathbf{a}_{u t}\left(\theta_{\mathrm{Q}}+\xi_{\mathrm{Q}}\right)\right]$ can be regarded as the sum of two matrices.

$$
\mathbf{Q}_{t}=\mathbf{Q}+\mathbf{\Theta} \boldsymbol{\Lambda},
$$

where $\Theta(\theta)=\left[\partial \mathbf{a}_{u}\left(\theta_{1}\right) / \partial \theta_{1}, \partial \mathbf{a}_{u}\left(\theta_{2}\right) / \partial \theta_{2}, \ldots, \partial \mathbf{a}_{u}\left(\theta_{Q}\right) / \partial \theta_{Q}\right]$ and $\Lambda=\operatorname{diag}(\xi)$.

Since the vectorized received signal $\mathbf{z}_{u}$ is only associated with DOA information, we construct the following function to verify the orthogonality:

$$
P=\max \sqrt{\left|\mathbf{Q}\left(\theta_{i}\right) \mathbf{z}_{u}\right|^{2}+\left|\boldsymbol{\Theta}\left(\theta_{i}\right) \mathbf{z}_{u}\right|^{2}}, \quad i=1,2, \ldots, \mathbf{Q} .
$$

When $\theta_{i}+\xi_{i}$ approaches the true incident angle, (28) achieves a peak value. Compared with (27), (28) still has orthogonality without $\Lambda$ because the vectorized received signal can also be represented as

$$
\mathbf{z}_{u}=(\mathbf{Q}+\boldsymbol{\Theta} \operatorname{diag}(\boldsymbol{\xi})) \mathbf{H}_{s}^{T}=\mathbf{Q} \mathbf{H}_{s}^{T}+\boldsymbol{\Theta}\left(\boldsymbol{\xi} \oplus \mathbf{H}_{s}^{T}\right),
$$

where $\mathbf{H}_{s}^{T}$ denotes sparse signal vector with $k$ nonzero values. It can be indicated from (29) that if $\Theta$ is orthogonal to the $q$ - th element $\mathbf{H}_{s}^{T}[q]$, it is also orthogonal to $\left(\Lambda \mathbf{H}_{s}^{T}\right)^{[q]}$, $q=1,2, \ldots, Q$.

After the first search around initial DOA estimates, we construct $\widetilde{A}=\left[\mathbf{a}_{u}\left(\theta_{m_{1}}\right), \partial \mathbf{a}_{u}\left(\theta_{m_{1}}\right) / \partial \theta_{m_{1}}\right]$ from $\mathbf{Q}$ and $\Theta$ corresponding to $\max (P)$. Thus, according to (29), leastsquares criterion is employed to estimate $\mathbf{H}_{s}^{T\left[m_{1}\right]}$ and $\left(\Lambda \mathbf{H}_{s}^{T}\right)^{\left[m_{1}\right]}$, which is expressed as

$$
\left[\begin{array}{c}
\mathbf{H}_{s}^{T}{ }^{\left[m_{1}\right]} \\
\left(\mathbf{\Lambda} \mathbf{H}_{s}^{T}\right)^{\left[m_{1}\right]}
\end{array}\right]=\left(\widetilde{A} \widetilde{A}^{H} \tilde{A} \tilde{A}\right)^{-1} \tilde{A} \widetilde{A}^{H} \mathbf{z}_{u} .
$$

The other $K-1$ signals are also measured by (28). In particular, the rows in $\mathbf{z}_{u}$ corresponding to $\theta_{m_{1}}, \theta_{m_{2}}, \cdots, \theta_{m_{k}}$ which have been estimated should be removed. Hence, the virtual received signal $\mathbf{z}_{u}$ is updated: 


$$
\mathbf{z}_{u}=\mathbf{z}_{u}-\sum_{i=1}^{k}\left[\mathbf{a}_{u}\left(\theta_{m_{i}}\right), \frac{\partial \mathbf{a}_{u}\left(\theta_{m_{i}}\right)}{\partial \theta_{m_{i}}}\right]\left[\begin{array}{c}
\mathbf{H}_{s}^{T}\left[m_{i}\right] \\
\left(\Lambda \mathbf{H}_{s}^{T}\right)^{\left[m_{i}\right]}
\end{array}\right] .
$$

The maximum value in each search corresponds to an incident angle. Eliminating sparse signal vector $\mathbf{H}_{s}^{T}\left[m_{k}\right]$, $\xi_{m_{k}}, k=1,2, \ldots, K$ can be computed by (30). The accurate and ambiguity-free DOA estimates are obtained with $K$ iterations.

$$
\widehat{\theta}_{k, s t}=\theta_{m k}+\xi_{m_{k}}, k=1,2, \ldots, K \text {. }
$$

4.3. Polarization Estimation. Inspired from (5), we can also construct a cross-correlation covariance matrix among the 6 electric and magnetic components. We focus on two combinations: electric components of the $x$-axis and $z$-axis and magnetic components of the $x$-axis and $z$-axis.

$$
\mathbf{R}_{e x, e z}=\operatorname{Adiag}\left(\sigma_{1}^{2} \mathbf{s}_{e x, 1} \mathbf{s}_{e z, 1}^{*}, \sigma_{2}^{2} \mathbf{s}_{e x, 2} \mathbf{s}_{e z, 2}^{*}, \ldots, \sigma_{K}^{2} \mathbf{s}_{e x, K} \mathbf{s}_{e z, K}^{*}\right) \mathbf{A}^{H}
$$

$\mathbf{R}_{h x, e z}=\operatorname{Adiag}\left(\sigma_{1}^{2} \mathbf{s}_{h x, 1} \mathbf{s}_{e z, 1}^{*}, \sigma_{2}^{2} \mathbf{s}_{h x, 2} \mathbf{s}_{e z, 2}^{*}, \ldots, \sigma_{K}^{2} \mathbf{s}_{h x, K} \mathbf{s}_{e z, K}^{*}\right) \mathbf{A}^{H}$

Performing vectorization operation similar to Section 3.2 , (33) and (34) are transformed to

$$
\begin{aligned}
\mathbf{r}_{e x, e z} & =\mathbf{A}^{*} \odot \mathbf{A}\left[\sigma_{1}^{2} \mathbf{s}_{e x, 1} \mathbf{s}_{e z, 1}^{*}, \sigma_{2}^{2} \mathbf{s}_{e x, 2} \mathbf{s}_{e z, 2}^{*}, \ldots, \sigma_{K}^{2} \mathbf{s}_{e x, K} \mathbf{s}_{e z, K}^{*}\right]^{T} \\
& =\mathbf{A}^{*} \odot \mathbf{A} \mathbf{S}_{e x, e z}, \\
\mathbf{r}_{h x, e z} & =\mathbf{A}^{*} \odot \mathbf{A}\left[\sigma_{1}^{2} \mathbf{s}_{h x, 1} \mathbf{s}_{e z, 1}^{*}, \sigma_{2}^{2} \mathbf{s}_{h x, 2} \mathbf{s}_{e z, 2}^{*}, \ldots, \sigma_{K}^{2} \mathbf{s}_{h x, K} \mathbf{s}_{e z, K}^{*}\right]^{T} \\
& =\mathbf{A}^{*} \odot \mathbf{A} \mathbf{S}_{h x, e z},
\end{aligned}
$$

where $\mathbf{A}$ can be computed by DOA estimates. $\mathbf{S}_{e x, e z}=\left[\sigma_{1}^{2} \mathbf{s}_{e x, 1} \mathbf{s}_{e z, 1}^{*}, \sigma_{2}^{2} \mathbf{s}_{e x, 2} \mathbf{s}_{e z, 2}^{*}, \ldots, \sigma_{K}^{2} \mathbf{s}_{e x, K} \mathbf{s}_{e z, K}^{*}\right]^{T}$ and $\mathbf{S}_{h x, e z}$ $=\left[\sigma_{1}^{2} \mathbf{s}_{h x, 1} \mathbf{s}_{e z, 1}^{*}, \sigma_{2}^{2} \mathbf{s}_{h x, 2} \mathbf{s}_{e z, 2}^{*}, \ldots, \sigma_{K}^{2} \mathbf{s}_{h x, K} \mathbf{s}_{e z, K}^{*}\right]^{T}$. The crosscorrelation covariance matrices after vectorization operation still contain polarization information, which provides a basis for polarization estimation. Least-squares criterion is utilized as

$$
\begin{aligned}
& \mathbf{S}_{e x, e z}=\left(\mathbf{A}^{H} \mathbf{A}\right)^{+} \mathbf{A}^{H} \mathbf{r}_{e x, e z}, \\
& \mathbf{S}_{h x, e z}=\left(\mathbf{A}^{H} \mathbf{A}\right)^{+} \mathbf{A}^{H} \mathbf{r}_{h x, e z} .
\end{aligned}
$$

According to the definition in (1), auxiliary polarization angle and polarization phase difference estimates are obtained by eliminating the power of signals.

$$
\begin{aligned}
& \hat{\gamma}_{k, e s t}=\arctan \left[\operatorname{abs}\left(\frac{\mathbf{S}_{h x, e z}^{[k]}}{\mathbf{S}_{e x, e z}^{[k]}}\right)\right], \\
& \hat{\eta}_{k, e s t}=-\operatorname{angle}\left(\frac{\mathbf{S}_{e x, e z}^{[k]}}{\mathbf{S}_{h x, e z}^{[k]}}\right),
\end{aligned}
$$

where $k=1,2, \ldots, K$.
4.4. Discussion. The proposed algorithm aims to jointly estimate DOA and polarization parameters with low complexity. There are mainly two steps where computational burden is effectively reduced. The first is the DOA initialization during which only eigendecomposition approach is used. Thus, coarse initial DOA estimates are obtained. Benefitting from that, we require no global overcomplete dictionary for orthogonal verification. The second step is reducing the dimensions of overcomplete dictionary from three to one because the two polarization parameters are both eliminated by the construction of the new covariance matrix $\mathbf{R}$ and the process of OGOMP algorithm is only related to DOA. In addition, benefitting from OGOMP algorithm, not only is the accuracy guaranteed but also the search interval is not strictly required. When the incident angle is not involved in the dictionary, estimation performance can also be guaranteed.

The process of the DOA and polarization estimation is summarized as follows:

Step 1: compute the estimates of $\mathbf{A}_{s}$ by eigendecomposition of $\mathbf{R}_{s}$

Step 2: eliminate polarization information from $\mathbf{A}_{s}$ with vector cross-product estimator

Step 3: obtain coarse DOA initial estimates in normalized Poynting vector

Step 4: search in partly overcomplete dictionary with OGOMP algorithm for accurate DOA estimates

Step 5: construct cross-correlation covariance matrix, perform vectorization operation, and obtain polarization estimates by least-square criterion

The main complexity of the proposed algorithm is discussed as follows.

Constructing the covariance matrix requires $O\left\{J(6 P)^{2}+8 J P^{2}\right\}$. The computational burden of DOA initialization involves the eigendecomposition which takes $O\left\{(6 P)^{3}+2 K^{3}\right\}$. OGOMP algorithm is composed of the search over the one-dimensional dictionary for $K$ iterations, which mainly requires $O\left\{K 2 Q\left|D_{u}\right|\right\}$. In summary, the proposed algorithm approximately needs the complexity of only $O\left\{J(6 P)^{2}+8 J P^{2}+(6 P)^{3}+2 K^{3}+K 2 Q\left|D_{u}\right|\right\}$. However, the traditional search-based algorithms cannot avoid three-dimensional search for three unknown parameters and the search range is relatively large compared with the proposed algorithm, which is exhaustive and infeasible.

\section{Simulation Results}

We perform some simulations in order to confirm the superior performance of the proposed SNA and the OGOMPbased algorithm. This section is divided into 4 parts. Part A verifies the large DOFs of SNA by scatter plot. Part B demonstrates the effectiveness of SF versus SNR and snapshots. We also compare the proposed algorithm with OMP, ESPRIT, and PM algorithms to outstand the prominent performance of the proposed algorithm in part C. Moreover, considering the different coupling leakage in different arrays, part $\mathrm{D}$ simulates the performance for 


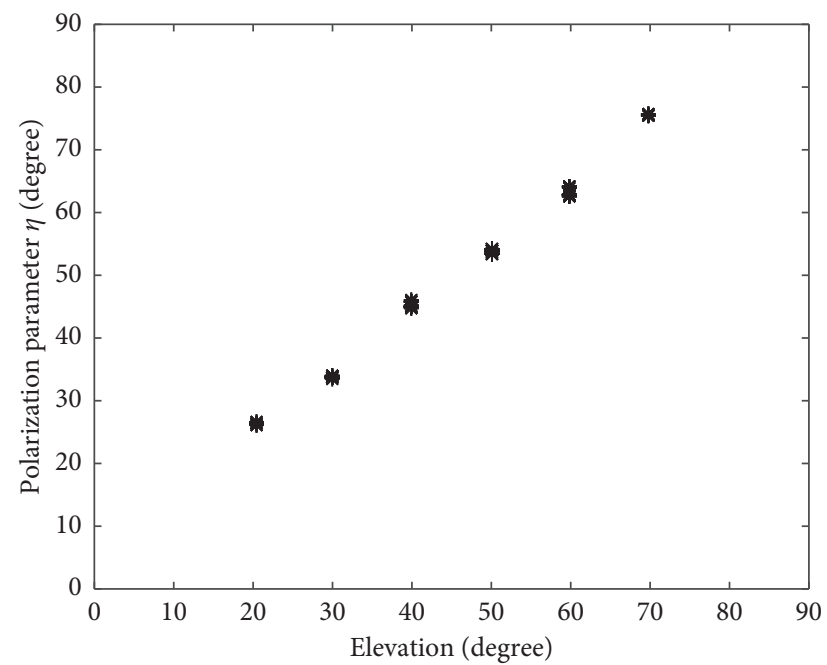

Figure 6: Scatter plot of $\theta$ and $\eta$ estimation results.

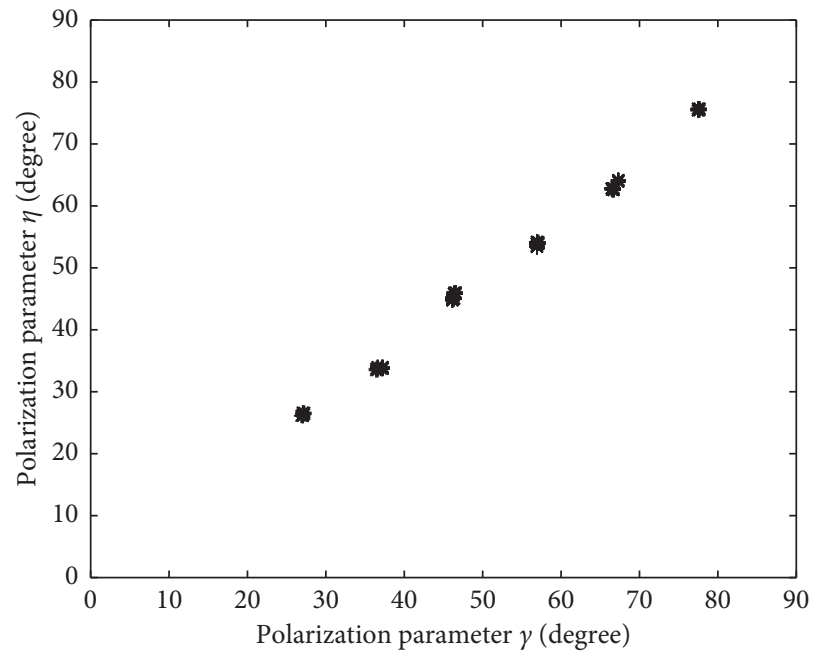

FIgURE 7: Scatter plot of $\gamma$ and $\eta$ estimation results.

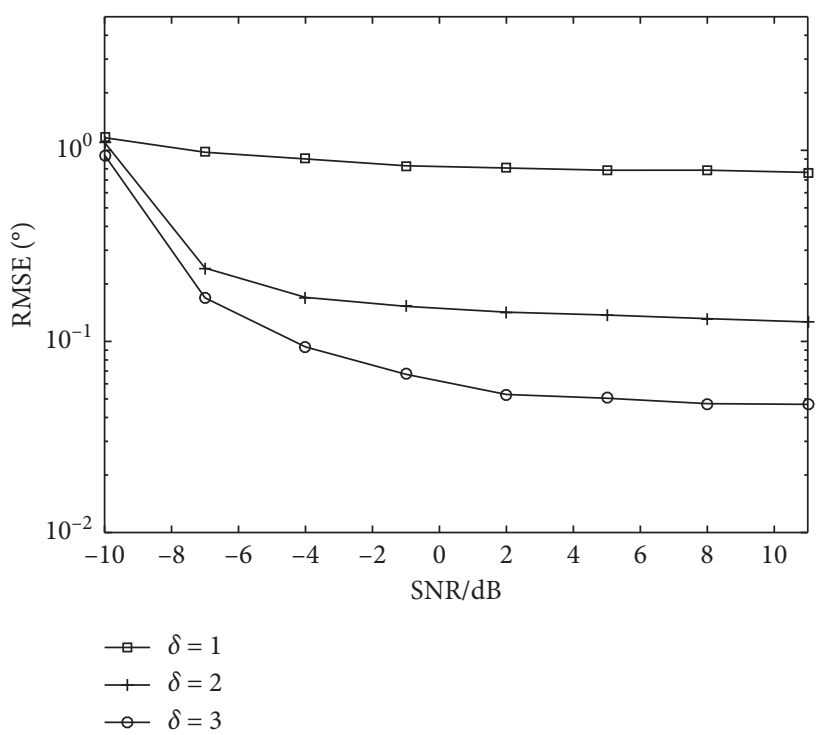

FIGURE 8: DOA estimation performance versus SF (SNR). 


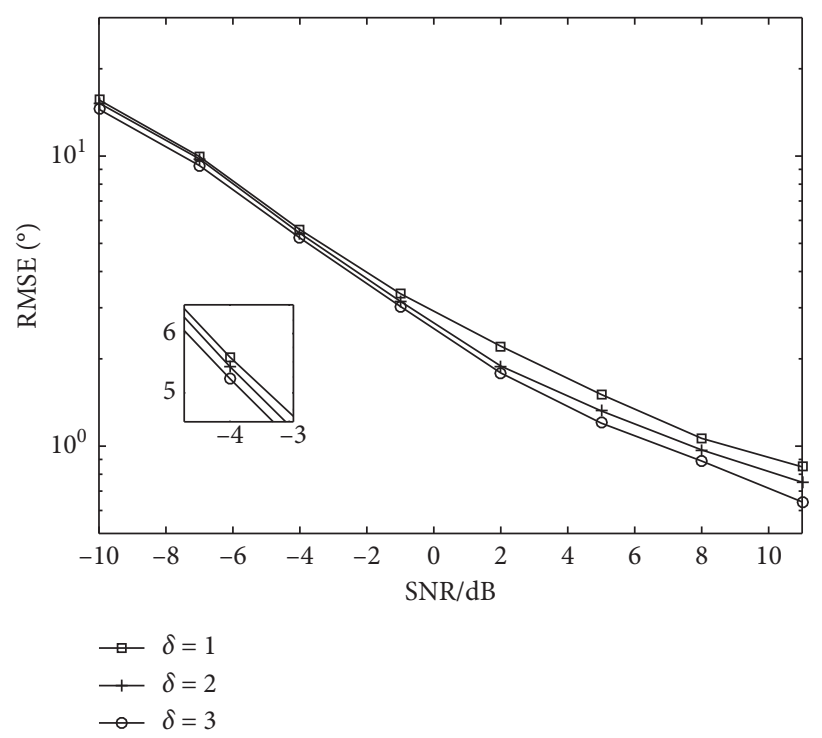

Figure 9: $\gamma$ estimation performance versus SF (SNR).

different array structures with mutual coupling effect. During the above simulations, root mean square error (RMSE) is employed to evaluate the performance, which is defined as

$$
\begin{aligned}
& \operatorname{RMSE}_{a}=\frac{1}{K} \sum_{k=1}^{K} \sqrt{\frac{1}{I} \sum_{i=1}^{I}\left[\left(\widehat{\theta}_{k, i}-\theta_{k}\right)^{2}\right]}, \\
& \operatorname{RMSE}_{\gamma}=\frac{1}{K} \sum_{k=1}^{K} \sqrt{\frac{1}{I} \sum_{i=1}^{I}\left[\left(\widehat{\gamma}_{k, i}-\gamma_{k}\right)^{2}\right]}, \\
& \operatorname{RMSE}_{\eta}=\frac{1}{K} \sum_{k=1}^{K} \sqrt{\frac{1}{I} \sum_{i=1}^{I}\left[\left(\hat{\eta}_{k, i}-\eta_{k}\right)^{2}\right]},
\end{aligned}
$$

where $\widehat{\theta}_{k, i}, \widehat{\gamma}_{k, i}$, and $\widehat{\eta}_{k, i}$ are the estimated values of $\theta_{k}, \gamma_{k}$, and $\eta_{k}$ during the $i$-th simulation, and $I$ is the number of independent simulations. In this paper, we assume that signal number $K$ has been estimated and there are 3 signals impinging on the array in simulation parts $B, C$, and $D$ with DOA and polarization parameters $\left(\theta_{1}, \gamma_{1}, \eta_{1}\right)=\left(20^{\circ}\right.$, $\left.27^{\circ}, 25^{\circ}\right), \quad\left(\theta_{2}, \gamma_{2}, \eta_{2}\right)=\left(30^{\circ}, 37^{\circ}, 35^{\circ}\right), \quad$ and $\left(\theta_{3}, \gamma_{3}, \eta_{3}\right)=$ $\left(40^{\circ}, 57^{\circ}, 55^{\circ}\right)$.

5.1. Independent DOA and Polarization Estimation. As is analyzed in Section 3, the proposed algorithm can estimate signals equal to or more than physical sensor elements benefitting from the SNA array structure and the vectorization operation. Figures 6 and 7 exhibit the scatter plot of the estimate pairs with 100 independent experiments. The physical sensor elements are 5 with array structure SNA $\lfloor 2,1,3,3,2\rfloor$, whereas there are 6 signals with DOA and polarization parameters $\left(\theta_{1}, \gamma_{1}, \eta_{1}\right)=\left(20^{\circ}, 27^{\circ}, 25^{\circ}\right),\left(\theta_{2}, \gamma_{2}, \eta_{2}\right)=\left(30^{\circ}, 37^{\circ}\right.$, $\left.35^{\circ}\right), \quad\left(\theta_{3}, \gamma_{3}, \eta_{3}\right)=\left(40^{\circ}, 47^{\circ}, 45^{\circ}\right), \quad\left(\theta_{4}, \gamma_{4}, \eta_{4}\right)=\left(50^{\circ}, 57^{\circ}\right.$,

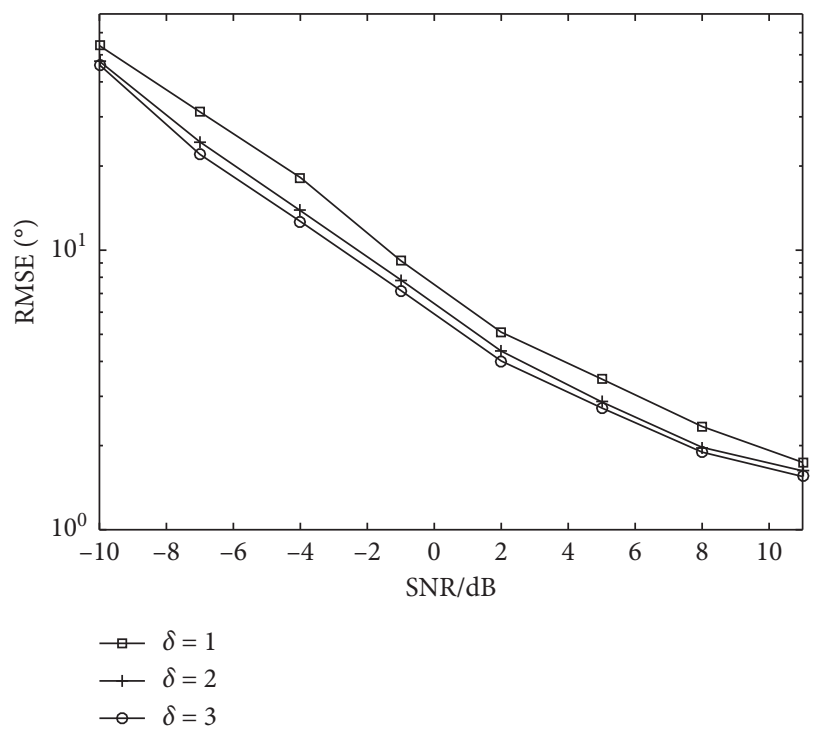

FIGURE 10: $\eta$ estimation performance versus SF (SNR).

$\left.55^{\circ}\right),\left(\theta_{5}, \gamma_{5}, \eta_{5}\right)=\left(60^{\circ}, 67^{\circ}, 65^{\circ}\right)$, and $\left(\theta_{6}, \gamma_{6}, \eta_{6}\right)=\left(60^{\circ}, 77^{\circ}\right.$, $\left.75^{\circ}\right)$, where $J=1000$ and $\mathrm{SNR}=20 \mathrm{~dB}$. As is depicted in the figures, all 6 signals are estimated accurately without any missing or error signal, which verifies advantages of large DOFs. Besides, the DOA and the two polarization parameters are autopaired, indicating that no extra pairing operation is needed.

5.2. Parameter Estimation Performance Comparison versus $S F$. In this part, we perform the SF simulation for SNA $\lfloor 3,1,3,4, \delta\rfloor$ with the proposed algorithm. Figures 8-10 demonstrate the RMSE performance for DOA and polarization parameters along with SNR, where $J=200$, and Figures 11-13 depict the RMSE performance based on different snapshots, where SNR $=0 d B$. As is revealed in the 


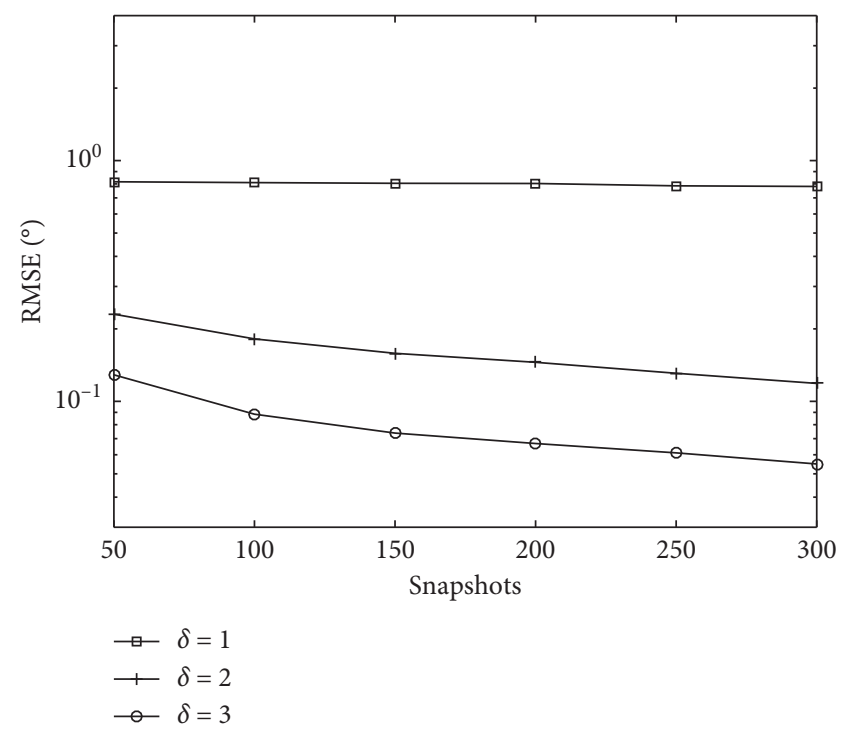

FIGURE 11: DOA estimation performance versus SF (snapshots).

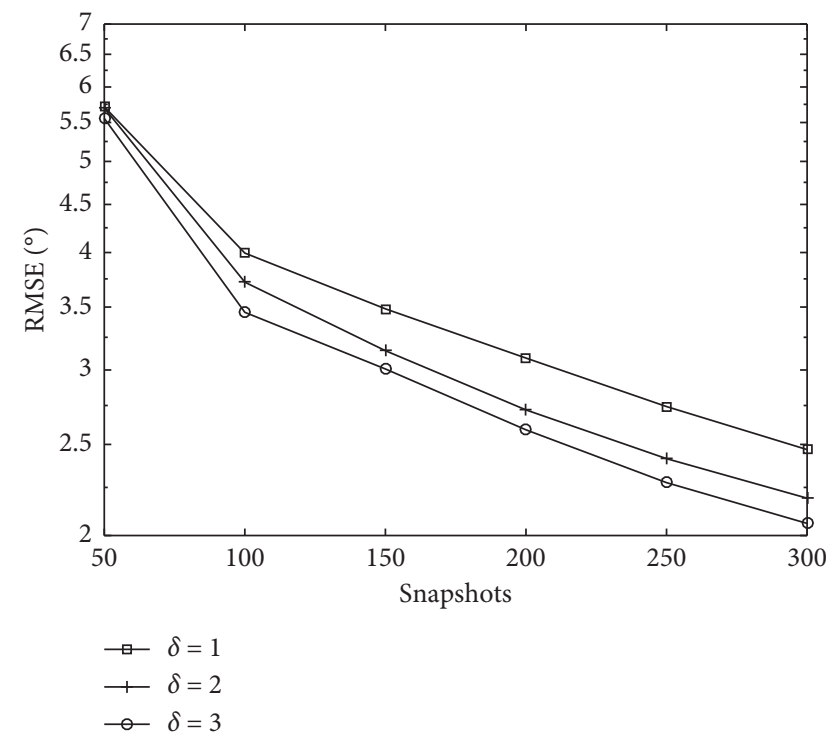

FIGURE 12: $\gamma$ estimation performance versus SF (snapshots).

figures, RMSE decreases not only with the improvement of SNR and snapshots but also accompanied by the enlargement of SF. From the overall perspective, the RMSE of DOA is much lower than the two polarization parameters.

5.3. Parameter Estimation Performance Comparison for Algorithms. Figures 14-16 compare the proposed algorithm with OMP, Propagator Method (PM) [28], and Estimating Signal Parameter via Rotational Invariance Techniques (ESPRIT), where snapshots $J=200$ and SNR $=[-10,11] d B$. In addition, Cramér-Rao Bound (CRB) [29] is presented as the standard.

The array structure is SNA $\lfloor 3,1,3,4,2\rfloor$. The dictionary intervals are all $0.1^{\circ}$ for OGOMP, OMP, and PM algorithms.

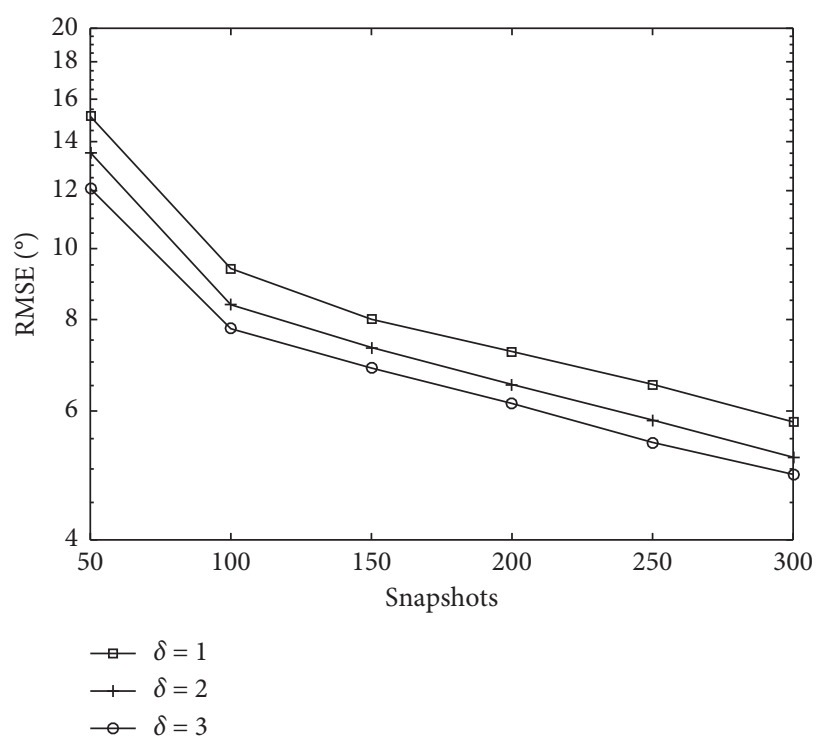

FIGURE 13: $\eta$ estimation performance versus SF (snapshots).

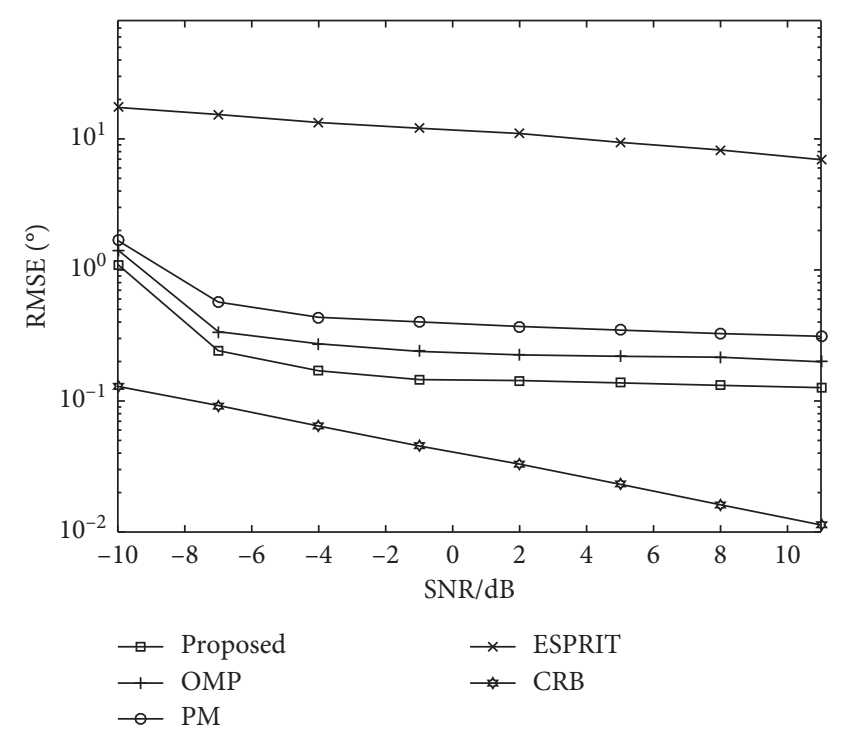

FIgURE 14: DOA estimation performance under different algorithms.

As revealed in the figures, the proposed algorithm outperforms the other three algorithms. Specifically, OMP algorithm ignores any possible incident angle outside the dictionary, which degrades the performance. PM requires no eigendecomposition but employs the search function with poor orthogonality. ESPRIT performs the worst because there are not enough sensors so that signal subspace matrix has insufficient information for DOA estimates. Simultaneously, the polarization estimates are also affected.

5.4. Parameter Estimation Performance Comparison for Array Structures. Since mutual coupling effect is varying for different array structures, we consider the coupling leakage in 


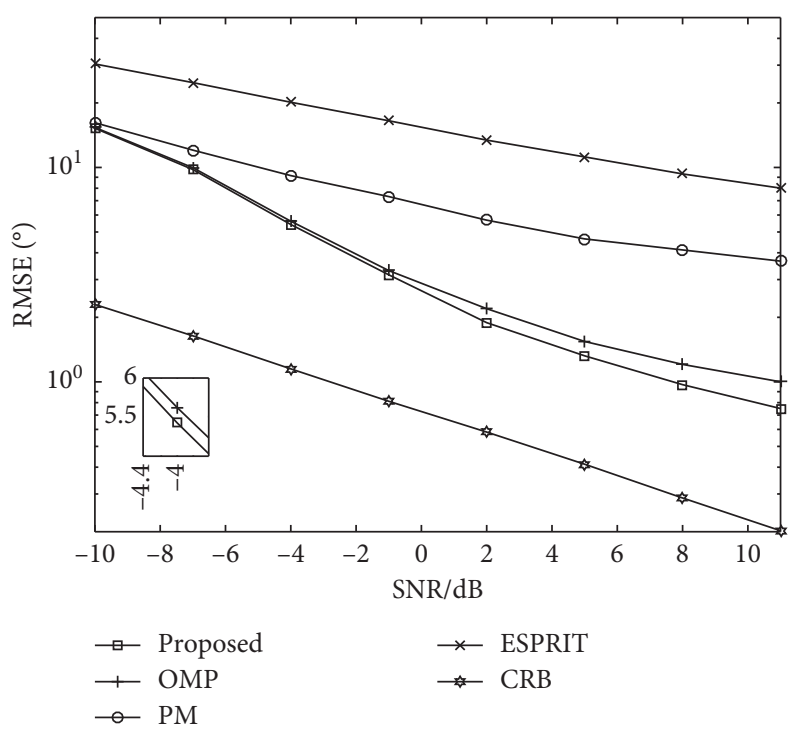

FIGURE 15: $\gamma$ estimation performance under different algorithms.

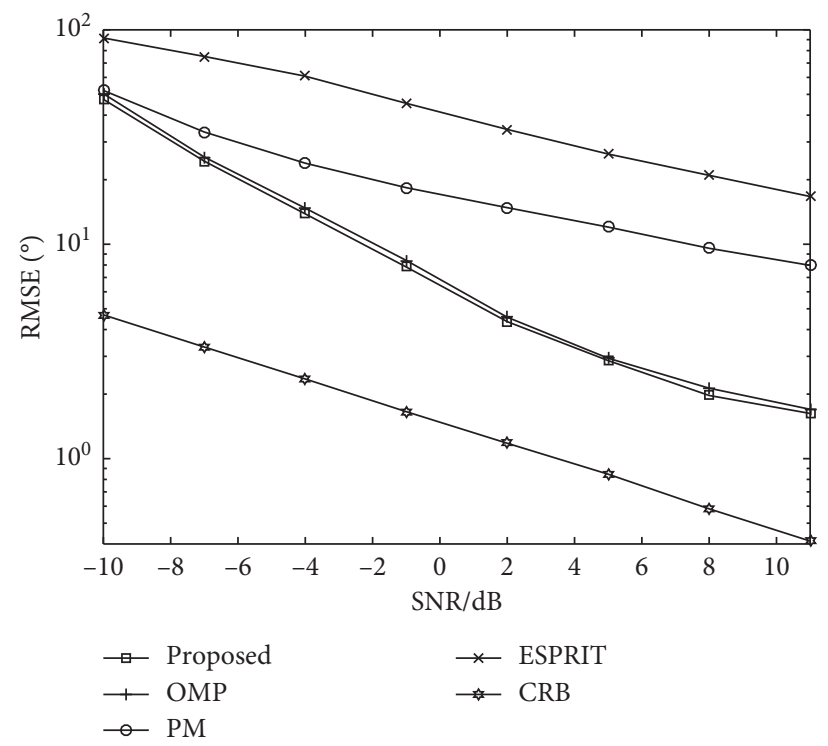

FIGURE 16: $\eta$ estimation performance under different algorithms.

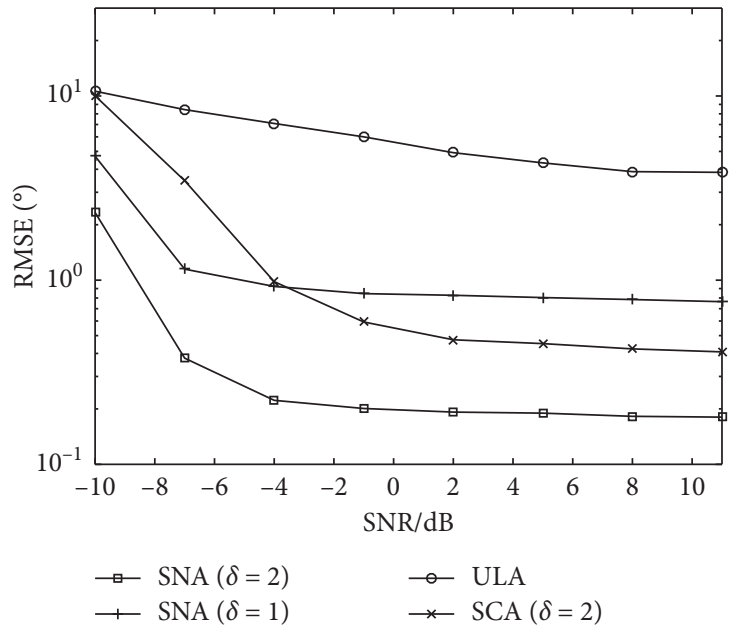

FIGURE 17: DOA estimation performance with different array structures. 


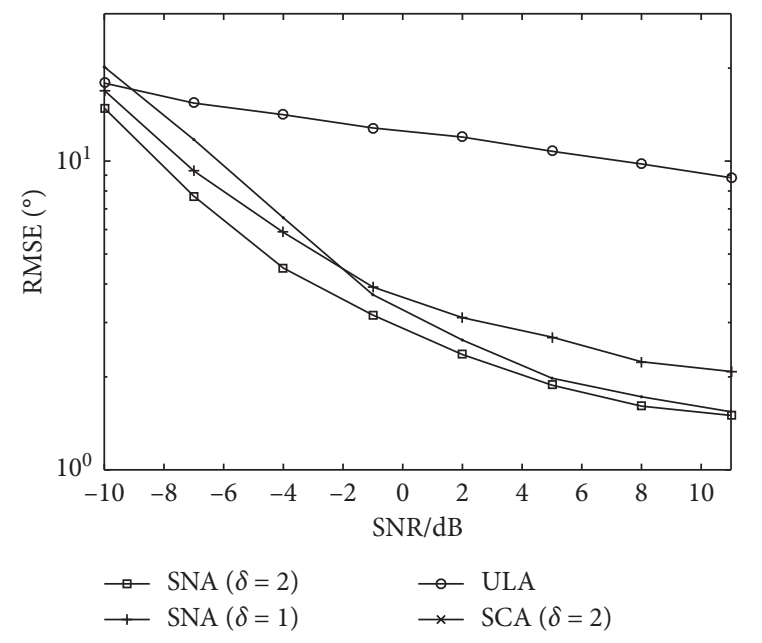

FIgURE 18: $\gamma$ estimation performance with different array structures.

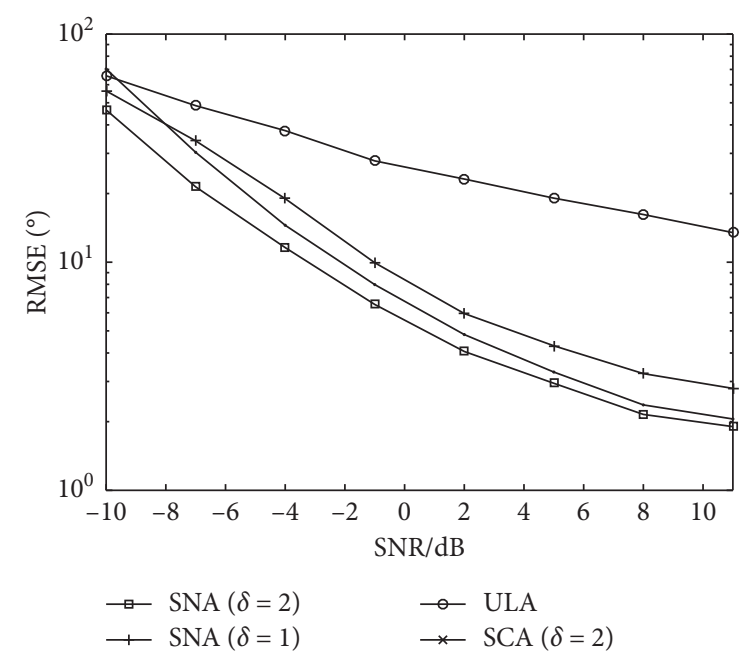

FIGURE 19: $\eta$ estimation performance with different array structures.

the simulation of array structure performance. The proposed algorithm is tested where $J=200$ and SNR $=[-10,11] d B$. The 4 array structures all have 6 sensors: SNA $[3,1,3,4,1\rfloor$, SNA $\lfloor 3,1,3,4,2\rfloor$, ULA $(d=\lambda / 2)$, and SCA $\lfloor 4,3,3,4,2\rfloor$.

As Figures 17-19 reveal, SNA $\lfloor 3,1,3,4,2\rfloor$ acts best due to its large DOFs and array aperture. Although SCA $\lfloor 4,3,3,4,2\rfloor$ is a sparse array, the missing elements in virtual array are the main cause of performance deterioration.

\section{Conclusion}

In this paper, a new array structure of sparse nested array is constructed, and a low-complexity off-grid orthogonal matching pursuit (OGOMP) algorithm is designed based on the proposed array structure to obtain DOA and polarization estimates. By introducing the sparse factor in EVSA, the proposed array structure has low mutual coupling, flexible array aperture, and high achievable degrees of freedom.
Benefitting from the DOA initialization and polarization parameter elimination, the overcomplete dictionary is compact to tremendously reduce computational complexity. Meanwhile, the proposed OGOMP algorithm searches for the unambiguous high-precise DOA estimates and solves the problem of poor performance for off-grid signals in OMP algorithm. Finally, polarization estimates are measured by cross-correlation covariance matrix and estimated directional matrix based on least-square criterion.

\section{Data Availability}

The data used to support the findings of this study are available from the corresponding author upon reasonable request.

\section{Conflicts of Interest}

The authors declare that they have no conflicts of interest.

\section{Acknowledgments}

This work was supported by China NSF (Grants 61971217, 61971218, and 61631020), Jiangsu NSF (Grant BK20200444), the fund of Sonar Technology Key Laboratory (research on the theory and algorithm of signal processing for two-dimensional underwater acoustics coprime array), the fund of Sonar Technology Key Laboratory (range estimation and location technology of passive target via multiple array combination), and Jiangsu Key Research and Development Project (BE2020101).

\section{References}

[1] A. Abdi and H. H. Guo, "Signal correlation modeling in acoustic vector sensor arrays," IEEE Transactions on Signal Processing, vol. 57, no. 3, pp. 892-903, 2009.

[2] X. Zhang, W. Liu, Y. Xu, and Z. Liu, "Quaternion-valued robust adaptive beamformer for electromagnetic vectorsensor arrays with worst-case constraint," Signal Processing, vol. 104, pp. 274-283, 2014.

[3] B. Li and Y. X. Zou, "Improved DOA estimation with acoustic vector sensor arrays using spatial sparsity and subarray manifold," in Proceedings of the IEEE International Conference on Acoustics. IEEE, pp. 2557-3560, Kyoto, Japan, March 2012.

[4] G. Zheng, "Two-dimensional DOA estimation for polarization sensitive array consisted of spatially spread crossed-dipole," IEEE Sensors Journal, vol. 18, no. 12, pp. 5014-5023, 2018.

[5] K. T. Wong, Y. Song, C. J. Fulton, S. Khan, and W.-Y. Tam, "Electrically "long" dipoles in a collocated/orthogonal triadfor direction finding and polarization estimation," IEEE Transactions on Antennas and Propagation, vol. 65, no. 11, pp. 6057-6067, 2017.

[6] W. Si, P. Zhao, Z. Qu, and L. Wang, "Computationally efficient angle and polarization estimation in the presence of multipath propagation using dual-polarization vector sensor array," International Journal of Antennas and Propagation, vol. 2016, Article ID 7537160, 15 pages, 2016.

[7] J. Li and R. T. Compton, "Angle and polarization estimation using ESPRIT with a polarization sensitive array," IEEE Transactions on Antennas and Propagation, vol. 39, no. 9, pp. 1376-1383, 1991. 
[8] J. Li and R. T. Compton, "Two-dimensional angle and polarization estimation using the ESPRIT algorithm," IEEE Transactions on Antennas and Propagation, vol. 40, no. 5, pp. 550-555, 1992.

[9] Q. W. Yuan, Q. Chen, and K. Sawaya, "MUSIC based DOA finding and polarization estimation using USV with polarization sensitive array antenna," in Proceedings of IEEE Radio and Wireless Symposium, pp. 339-342, San Diego, CF, USA, October 2006.

[10] L. Wang, L. Yang, G. Wang, Z. Chen, and M. Zou, "Univector-sensor dimensionality reduction MUSIC algorithm for DOA and polarization estimation," Mathematical Problems in Engineering, vol. 2014, Article ID 682472, 9 pages, 2014.

[11] H. Chen, W. Wang, and W. Liu, "Joint DOA, range, and polarization estimation for rectilinear sources with a COLD array," IEEE Wireless Communications Letters, vol. 8, no. 5, pp. 1398-1401, 2019.

[12] K. T. Wong and M. D. Zoltowski, "High accuracy 2D angle estimation with extended aperture vector sensor arrays," in Proceedings of the IEEE International Conference on Acoustics, Atlanta, GA, USA, May 1996.

[13] K. T. Wong and M. D. Zoltowski, "Uni-vector-sensor ESPRIT for multisource azimuth, elevation, and polarization estimation," IEEE Transactions on Antennas and Propagation, vol. 45, no. 10, pp. 1467-1474, 1997.

[14] K. T. Wong and M. D. Zoltowski, "Closed-form direction finding and polarization estimation with arbitrarily spaced electromagnetic vector-sensors at unknown locations," IEEE Transactions on Antennas and Propagation, vol. 48, no. 5, pp. 671-681, 2000.

[15] M. D. Zoltowski and K. T. Wong, "Closed-form eigenstructurebased direction finding using arbitrary but identical subarrays on a sparse uniform cartesian array grid," IEEE Transactions on Signal Processing, vol. 48, no. 8, pp. 2205-2210, 2000.

[16] C. Hou, C. Fang, Z. Deng, Y. Wang, and W. Si, "Two-dimensional direction-of-arrival and polarization parameter estimation using parallel co-prime polarization sensitive array," IEEE Access, vol. 8, no. 8, pp. 6566-6574, 2020.

[17] Y. Yang, X. Mao, G. Jiang, and Y. Wang, "Spatially separated nested vector-sensor array with reduced mutual coupling," IEEE Sensors Journal, vol. 19, no. 14, pp. 5801-5817, 2019.

[18] W. Si, Y. Wang, and C. Zhang, "Three-parallel co-prime polarization sensitive array for 2-D DOA and polarization estimation via sparse representation," IEEE Access, vol. 7, pp. 15404-15413, 2019.

[19] N. Rashmi and M. Sarvagya, "Sparse channel estimation using orthogonal matching Pursuit algorithm for SCM-OFDM system," International Conference on Advances in Computing, pp. 1224-1227, 2016.

[20] G. A. Deschamps, “Techniques for handling elliptically polarized waves with special reference to antennas: part II-geometrical representation of the polarization of a plane electromagnetic wave," Proceedings of the IRE, vol. 39, no. 5, pp. 540-544, 1951.

[21] K. T. Wong, L. S. Linshan Li, and M. D. Zoltowski, "RootMUSIC-based direction-finding and polarization estimation using diversely polarized possibly collocated antennas," IEEE Antennas and Wireless Propagation Letters, vol. 3, no. 1, pp. 129-132, 2004.

[22] W. Zheng, X. Zhang, Y. Wang, M. Zhou, and Q. Wu, "Extended coprime array configuration generating large-scale antenna co-array in massive MIMO system," IEEE Transactions on Vehicular Technology, vol. 68, no. 8, pp. 7841-7853, 2019.
[23] P. Pal and P. P. Vaidyanathan, "Nested Arrays: a novel approach to array processing with enhanced degrees of freedom," IEEE Transactions on Signal Processing, vol. 58, no. 8, pp. 4167-4181, 2010.

[24] C. Zhou, Y. Gu, X. Fan, Z. Shi, G. Mao, and Y. D. Zhang, "Direction-of-arrival estimation for coprime array via virtual array interpolation," IEEE Transactions on Signal Processing, vol. 66, no. 22, pp. 5956-5971, 2018.

[25] M. D. Zoltowski and K. T. Wong, "ESPRIT-based 2-D direction finding with a sparse uniform array of electromagnetic vector sensors," IEEE Transactions on Signal Processing, vol. 48, no. 8, pp. 2195-2204, 2000.

[26] L. Zhang, W. Liu, and J. L. Richard, "A minimum variance beamformer with linear and quadratic constraints based on uniform linear antenna arrays," in Proceedings of the IEEE Antennas and Propagation Conference, pp. 585-588, Charleston, Soouth Carolina, June 2009.

[27] J. Li, Y. Li, and X. Zhang, "Two-dimensional off-grid DOA estimation using unfolded parallel coprime array," IEEE Communications Letters, vol. 22, no. 12, pp. 2495-2498, 2018.

[28] N. Tayem and H. M. Kwon, "L-shape 2-dimensional arrival angle estimation with propagator method," IEEE Transactions on Antennas and Propagation, vol. 53, no. 5, pp. 1622-1630, 2005.

[29] P. Stoica and A. Nehorai, "Performance study of conditional and unconditional direction-of-arrival estimation," IEEE Transactions on Acoustics, Speech, and Signal Processing, vol. 38, no. 10, pp. 1783-1795, 1990. 